DOI: http://dx.doi.org/10.22198/rys.2018.72.a904

Artículos

\title{
Menores migrantes de retorno: problemática académica y proceso administrativo en el sistema escolar sonorense
}

\author{
Returning migrant minors: academic problems \\ and administrative process in the Sonoran school system
}

\author{
Gloria Ciria Valdéz Gardea* \\ orcid.org/0000-0003-0064-815X \\ Liza Fabiola Ruiz Peralta** \\ orcid.org/0000-0002-3457-5326 \\ Oscar Bernardo Rivera García* \\ orcid.org/0000-0003-1557-3170 \\ Ramiro Antonio Iópez*** \\ orcid.org/0000-0002-9926-8888
}

Resumen: en este artículo se analizan las consecuencias de la migración internacional en los procesos académicos y admi-

\footnotetext{
* Autora para correspondencia. Doctora en antropología y profesora-investigadora de El Colegio de Sonora. Obregón \#54, colonia Centro, C. P. 83000 . Hermosillo, Sonora, México. Teléfono: (662) 259 5300. Correo electrónico: gvaldez@colson.edu.mx

** Maestra en ciencias sociales y estudiante de doctorado en la Universidad de Sonora. Blvd. Luis Encinas y Rosales s/n, colonia Centro, C. P. 83000 . Hermosillo, Sonora, México. Teléfono: (662) 111 5786. Correo electrónico: ruiz.fabiola@hotmail.com

*** Profesor-investigador en el Instituto de Investigaciones Sociales de la Universidad Autónoma de Baja California. Blvd. Benito Juárez s/n, colonia Insurgentes Este, C. P. 21280. Mexicali, Baja California, México. Teléfono: (662) 325 6091. Correo electrónico: rigo140479@yahoo. com.mx

***** Doctorante en ciencias sociales en El Colegio de Sonora. Berlín \#96, colonia Centenario Lux. C. P. 83278, Hermosillo, Sonora, México. Teléfono: (662) 195 0018. Correo electrónico: ramiantl@gmail.com
} 
nistrativos escolares, para ello se examinaron las funciones directivas en la inscripción de los menores migrantes de retorno a México. Los resultados se obtuvieron a partir de los datos recopilados en 34 escuelas primarias públicas de Hermosillo, Sonora, durante el ciclo escolar 2014-2015. Se aplicó una entrevista semiestructurada a 34 directores y a 3 funcionarios de la Secretaría de Educación y Cultura del estado, para identificar la dinámica administrativa que realizan al momento de recibir a los niños y las niñas con experiencia educativa en Estados Unidos, y conocer el grado de capacitación y conocimiento que tienen los directores, para facilitar el proceso de incorporación de los nuevos estudiantes al sistema educativo de Sonora.

Palabras clave: migración de retorno; niños inmigrantes; PROBEM; entrevistas.

Abstract: this article analyzes the consequences of international migration for the school academic and administrative processes; for this purpose, management functions in the enrollment of migrant minors returning to Mexico were examined. The results were obtained from data collected in 34 public primary schools in Hermosillo, Sonora during the 2014-2015 school year. A semi-structured interview was conducted with 34 school principals and three officials of the state Secretariat of Education and Culture in order to identify the administrative dynamics involved when receiving children with school experience in the United States, as well as to determine the level of training and knowledge of school principals with the aim of facilitating the process of incorporation of new students into Sonora's education system.

Keywords: return migration; immigrant children; PROBEM; interviews. 
Recibido el 24 de febrero de 2017. Aceptado el 5 de octubre de 2017.

\section{Introducción}

Las familias con estatus migratorio mixto, es decir, las integradas tanto por ciudadanos estadounidenses como por indocumentados, durante décadas han vivido en la incertidumbre ante la amenaza siempre presente de la deportación. Con la llegada de Donald Trump a la presidencia de Estados Unidos se ha elevado la preocupación e incertidumbre de las familias migrantes y de los grupos defensores de los derechos humanos. El presidente Trump basó su campaña en un discurso xenofóbico y una retórica nativista, así como en la promesa de deportar a millones de inmigrantes indocumentados, a quienes caracterizó como delincuentes y criminales. Las acciones ejecutivas que firmó durante sus primeras dos semanas en el gobierno, para autorizar la construcción de un muro entre la frontera de México y Estados Unidos y prohibir el ingreso de ciudadanos de siete países de mayoría musulmana, han prendido los focos de alerta, y contribuyen a aumentar la zozobra entre los inmigrantes (Ye Hee Lee 2015).

Según un estudio de Warren y Kerwin (2017), en 2014 en Estados Unidos había 3.3 millones de hogares habitados por familias con estatus migratorio mixto y 6.6 millones de individuos, nacidos en el país, que compartían el hogar con residentes indocumentados, 5.7 millones de ellos eran menores de 18 años. Estas estadísticas muestran la grave problemática que implica la deportación de inmigrantes indocumentados quienes, en la mayoría de los casos, traen consigo a sus hijos menores en edad escolar, lo cual puede representar un reto serio para los menores que desean continuar su educación en escuelas en México, y también para su sistema educativo.

Pero, ¿qué se entiende aquí por menores migrantes de retorno? La definición es operativa y su construcción se basa en resultados de estudios previos (Valdéz y García 2017; Valdéz 2012; 2011). Las características del retorno a Sonora se analizan de manera no lineal, como un proceso heterogéneo y familiar cuyos actores son niños y adolescentes nacidos o no en México, pertenecientes a una familia 
mixta que tuvo que volver voluntariamente o fue forzada a hacerlo. El análisis es no lineal porque las razones y las circunstancias del retorno varían: deportación, repatriación o decisión propia, sin haber pasado por un trámite administrativo. En muchas ocasiones su retorno es escalonado, y no necesariamente vuelven a la comunidad de origen y tampoco significa que sea definitivo. Se trata de un proceso heterogéneo porque puede ocurrir de una manera diferenciada dentro de las regiones de México, y es familiar porque la decisión del retorno no es individual, sino que la familia acuerda estrategias para planearlo con apoyo de las redes sociales en México o Estados Unidos, en especial cuando uno de los padres es deportado.

¿Cómo se habla de retorno, si algunos de estos menores nacieron en Estados Unidos y nunca se fueron de México? Esto se debe a que el fenómeno se analiza de manera familiar, porque se trata de hijos de padres mexicanos que vuelven a su país de origen. Esta conceptualización operativa ayuda a seguir trabajando en el concepto de menores migrantes de retorno, con las aportaciones de autores que se refieren a ellos como menores trasnacionales (Zúñiga et al. 2008), binacionales (Moctezuma 2013) o de retorno trasgeneracional (Durand 2004).

Los estudios sobre el retorno de menores migrantes y su incorporación al sistema educativo mexicano son recientes (Sánchez y Zúñiga 2010; Suárez-Orozco y Qin-Hilliard 2004; Zúñiga et al. 2008; Franco 2014; Silva y Cruz 2013), tienen una perspectiva multidisciplinaria, y su finalidad es exponer la situación que representa -tanto para el Estado, las instituciones educativas y la familia que regresa- la llegada de niños y niñas con experiencia educativa en otro país. En Sonora, el interés está enfocado en analizar aspectos socioacadémicos e institucionales, que convergen desde el momento en que una familia decide regresar a México, de manera voluntaria o no (Ruiz 2011; Ruiz y Valdéz 2012; Valdéz y García 2017; Valdéz 2011) y en los niños y los adolescentes que representan el grupo más vulnerable. Aquí se analiza el trámite administrativo que realizan las escuelas primarias públicas en Hermosillo, para inscribir a los menores migrantes de retorno con experiencia educativa en Estados Unidos. Una de las estrategias metodológicas principales se basó en la consulta de documentación oficial, como la norma de control escolar y forma- 
tos administrativos, así como testimonios de directores, supervisoras escolares y de la Dirección de Registro y Certificación de Educación Básica de la Secretaría de Educación y Cultura (SEC) de Sonora. Los hallazgos preliminares apuntan a que hay deficiencias en la capacitación del personal directivo, y se detectaron al menos tres tipos de inconsistencias en el discurso de los directores y el personal de dicha dependencia sobre los procesos de identificación de los alumnos y las cifras de los ingresos.

\section{Metodología}

Este artículo es el resultado de un análisis acumulativo de investigación llevado a cabo en Sonora, de 2011 a 2016, e intenta contribuir a la reflexión sobre el contexto situacional-educativo de los menores migrantes de retorno procedentes de EE UU. ${ }^{1}$ Se pretende dar cuenta del volumen de los alumnos con estas características, inscritos durante el ciclo escolar 2014-2015 y el primer bimestre de 20152016. El trabajo de campo se realizó en el primer bimestre del ciclo escolar 2015-2016 en Hermosillo. Se entrevistó a los directores, con la intención de conocer el número de alumnos migrantes de retorno procedentes de Estados Unidos que se inscribieron en el ciclo 20142015, y los ingresos presentados hasta ese momento.

Como caso de estudio, se problematizan las dinámicas administrativas de las escuelas primarias públicas de Hermosillo, en el proceso de inscripción de esos alumnos. Estudios previos han demostrado que la mayoría de los menores se inscriben en dichas escuelas ${ }^{2}$ y que en Hermosillo se reporta la mayor recepción de ellos (Ruiz y Valdéz 2012; Valdéz 2012).

1 Ante la complejidad del término "menor migrante de retorno" y "alumno procedente de Estados Unidos", y la falta de una definición clara, durante las entrevistas en trabajo de campo se hizo énfasis en que el foco de interés del estudio son los alumnos migrantes de retorno con experiencia educativa en Estados Unidos. Por lo anterior, para definir a la población de estudio, se les explicó a los directores que se trataba de alumnos llegados de dicho país, o con experiencia en escuelas estadounidenses.

2 Aunque hay niños y niñas que lo hacen en el sector privado, aquí sólo se contempla a los inscritos en el público. 
De acuerdo con el Programa Binacional de Educación Migrante (PROBEM), ${ }^{3}$ en el ciclo escolar 2014-2015 hubo 576 alumnos inscritos en el estado; de ellos, 519 en primaria, ${ }^{4} 98$ en Hermosillo, que representan 18 por ciento. Como Sonora tiene 72 municipios, en cada uno estarían inscritos 7.2, en promedio, en primaria, por lo que 18 por ciento es representativo para el estado (véase Figura 1).

A partir de la información del PROBEM, la investigación se concentró en 44 escuelas primarias del sector público, ${ }^{5}$ donde los alumnos inscritos fueron 58 .

Figura 1. Municipios de Sonora receptores de estudiantes migrantes procedentes de Estados Unidos, que ingresaron

a escuelas primarias en el ciclo 2014-2015

\begin{tabular}{|c|c|c|c|}
\hline Municipio & $\begin{array}{c}\text { Total de alumnos de } \\
\text { primaria y secundaria }\end{array}$ & $\begin{array}{c}\text { Alumnos de } \\
\text { primaria }\end{array}$ & $\begin{array}{c}\text { Alumnos de } \\
\text { primaria pública }\end{array}$ \\
\hline Hermosillo & 115 & 98 & 58 \\
\hline Nogales & 87 & 82 & 0 \\
\hline San Luis Río Colorado & 80 & 75 & 0 \\
\hline Cajeme & 52 & 51 & 0 \\
\hline Agua Prieta & 42 & 30 & 0 \\
\hline Puerto Peñasco & 29 & 29 & 0 \\
\hline Magdalena de Kino & 20 & 17 & 0 \\
\hline Caborca & 18 & 120 & 0 \\
\hline Otros & 133 & 519 & 58 \\
\hline Total & 576 & & \\
\hline
\end{tabular}

Fuente: elaboración propia, con base en las listas del PROBEM de 2015.

3 Según el PROBEM, la información de alumnos procedentes de Estados Unidos no considera el nivel preescolar, y los datos proporcionados sólo indican la estadística del ciclo 20142015: http://www.sepen.gob.mx/probem/

4 Sólo 57 alumnos se inscribieron en secundaria en el ciclo 2014-2015 en Sonora (PROBEM 2015).

5 El trabajo se realizó de manera interna, debido a que esta información no se especificaba en las bases de datos del PROBEM. La estrategia fue identificar las escuelas primarias públicas de Hermosillo en el directorio del Sistema Nacional de Información de Escuelas de la Secretaría de Educación Pública (SEP), y requirió una selección meticulosa de ellas: http://www.snie. sep.gob.mx/SNIESC/ 
Después de tomar la decisión de concentrar la investigación en el municipio de Hermosillo, fue necesario ubicar espacialmente las escuelas públicas. La primera estrategia metodológica fue asistir a un taller impartido por el Instituto Nacional de Estadística y Geografía (INEGI), ${ }^{6}$ para conocer la distribución de los planteles, lo cual permitió elaborar un mapa temático donde se identifica la zona geográfica, el turno y el subsistema - estatal o federal-al que pertenecen.

La segunda estrategia para identificar a los actores fue solicitar al PROBEM un listado de las escuelas públicas con ingresos de alumnos procedentes de Estados Unidos durante el ciclo 2014-2015; esta base de datos incluyó también a los menores; en Hermosillo había 44, con 58 alumnos de este tipo. Se visitaron 34 escuelas, y se entrevistó a sus directores, de septiembre a noviembre de 2015; 23 de ellas ofrecen clases en el turno matutino y 11 en el vespertino, y representan 80 por ciento del total de las reportadas. El primer hallazgo significativo fue que en las 34 escuelas se identificaron más alumnos migrantes de retorno inscritos, que los reportados por el PROBEM en todas.

Con la entrevista semiestructurada, dirigida a los directores, se obtuvo información relacionada con los procesos administrativos de inscripción para los alumnos que provienen de escuelas estadounidenses. Debido a la ambigüedad conceptual que implicaba el término "menor migrante de retorno" para los directores, se les explicó que se trata de niños y niñas nacidos(as) en México o en EE UU, que han cursado algunos ciclos escolares allá o todos sus estudios en ese país, y que fueron inscritos en las primarias de Hermosillo durante los ciclos 2014-2015 y 2015-2016. Se les comentó que el tema principal del proyecto de investigación es la migración de retorno a Sonora, y que de acuerdo con cifras oficiales del Sistema de Información, Control y Registro Escolar de Sonora (SICRES) ${ }^{7}$ y el PROBEM se identificó a Hermosillo como la ciudad con mayor representatividad de familias y menores migrantes de retorno.

6 Según el INEGI, hay 117 escuelas primarias públicas en Hermosillo: (www.inegi.org.mx/ monografias/informacion/son/economia/infraestructura.aspx?tem).

Este dato es importante para la segunda parte de esta investigación, dado que interesa cotejar si en las escuelas que el PROBEM no reporta en sus bases de datos, hay niños y niñas migrantes procedentes de Estados Unidos.

7 www.sicres/hindex.aspx 
La entrevista incluyó 48 preguntas divididas en los temas siguientes: a) identificación de la escuela y años de servicio del director; b) cantidad de alumnos procedentes de Estados Unidos inscritos en el ciclo 2014-2015 y a la fecha de la entrevista; ${ }^{8}$ c) consideraciones, valoración y capacitación directiva de los servicios administrativos durante la inscripción de los migrantes y d) la existencia y conocimiento de alguna evaluación diagnóstica para el ingreso de un alumno con experiencia educativa en EE UU.

Con el mismo guion se entrevistó a tres funcionarios de áreas administrativas de la SEC: una supervisora escolar, personal de la Dirección de Registro y Certificación de Educación Básica y al coordinador estatal del Programa Binacional de Educación Migrante, dentro del área de asuntos internacionales. La finalidad de estas visitas fue conocer las funciones de estos departamentos en relación con la capacitación directiva, el conocimiento y flujo de información a directores sobre acuerdos y normas oficiales de la SEP, y la participación de estas instancias en la inscripción de niños y niñas migrantes.

\section{"Son como niños que están y no están": el discurso de los directores}

El fenómeno migratorio que experimentan las familias mexicanas que están regresando a México, en particular a Hermosillo, tiene efectos colaterales que evidencian su desconocimiento del sistema educativo, que homogeneiza a los estudiantes, y cuyo proceso de inscripción presenta contradicciones y ambigüedades que repiten los directores responsables de llevarlo a cabo. Es necesario que dicho sistema reconozca las diferencias culturales, sociales, familiares y económicas entre los/as niñas que provienen de escuelas estadounidenses. En especial si se toma en cuenta que algunas de las familias se ven forzadas a regresar a su país de origen debido a la crisis económica, la falta de documentación para trabajar o vivir en EE UU, la militarización de las fronteras, algún deceso o una repatriación, entre otras razones. Un

8 Una de las preguntas solicitaba la cantidad de alumnos procedentes de EE UU inscritos hasta ese momento, que consideraba las inscripciones durante el ciclo 2015-2016. 
retorno migratorio produce un impacto inmediato en la vida de los niños, que se debería considerar al momento de ingresar a las escuelas, sobre todo cuando es producto de una deportación, y no hubo oportunidad de recuperar documentación oficial para las inscripciones escolares.

En las respuestas, los 34 directores mencionaron dos requisitos mínimos para recibir a un niño en una escuela primaria de Hermosillo: una boleta de calificaciones o documento de trasferencia, expedido por la escuela donde cursó el último grado en EE UU, y el acta de nacimiento -puede ser mexicana o extranjera-. Uno de los directores expresó que "la educación no se le niega a nadie, se recibe al niño y se le trata exactamente como a cualquier otro alumno de la escuela primaria $[\ldots]$ ". Y la mayor parte de ellos comentaron que "[...] el sistema es muy noble porque no le pone ninguna traba para que los niños estudien aquí, no importa que sea extranjero, sonorense o de cualquier otro estado de la república".

En apariencia, cualquier niño/a se debe recibir, y de inmediato ubicarlo/a en el grado que le corresponde -después de haber comprobado sus estudios anteriores-. En teoría, cada director, por norma, debe cerciorarse de que todos los alumnos queden debidamente inscritos ante la SEC y en el portal del SICRES; esto es de suma importancia, porque si no "aparece" en el sistema, tampoco estará en las listas de asistencia. Una vez que el niño está "dado de alta" y el registro aparece en el portal del SICRES, se da por hecho que está formalmente inscrito en el sistema y entra en una homologación institucional, es decir, que se le tratará igual que a cualquier otro; llevará el mismo plan de estudios para el grado respectivo, la atención de los profesores y del director será la misma, así como sus obligaciones y responsabilidades. Esto admite que se reproduzca el discurso de homogeneizar al alumno, sin considerar el elemento situacional: su condición de ser menor migrante de retorno y provenir de un sistema educativo diferente.

En el sistema de educación no existe un parámetro o concepto preciso para identificar a los niños con experiencia migratoria en EE UU o cualquier otro país. Si el padre, madre o tutor al momento de la inscripción entrega un acta de nacimiento que compruebe que nació en otro país, se le considerará "alumno extranjero”, incluso si cursó 
toda su primaria en México. Se mantienen en la invisibilidad los/ as niñas nacidas en México, con acta de nacimiento nacional, pero que por diferentes circunstancias y periodos migraron desde o hacia Estados Unidos, y cursaron parte de su educación en dicho país. Su experiencia educativa no es considerada al momento de ser inscritos con el acta de nacimiento mexicana. Según los directores, parte de esta problemática se debe a que estas particularidades académicas y administrativas no se explican en la norma de control escolar, ni se registran en algún apartado del SICRES.

Para 83 por ciento de los directores, los documentos obligatorios que los/as niñas procedentes de EE UU deben presentar para ingresar son la boleta de calificaciones del grado anterior y el acta de nacimiento -mexicana o extranjera-. Dos mencionaron que sólo necesitan el documento de trasferencia; otro más dijo que con el acta de nacimiento era suficiente. Después de presentar los documentos se captura la información en el portal electrónico del SICRES, y así el niño queda inscrito. Los directores comentaron que al tratarse de una plataforma "virtual", ellos no pueden inscribir a los estudiantes de otro estado de la república o de otro país. Cuando se les preguntó quién inscribe en el portal de SICRES a los alumnos procedentes de EE UU, dijeron que el personal de la Dirección de Registro y Certificación de Educación Básica; 80 por ciento de ellos expresó que dicha dirección maneja al SICRES, y que a ellos se les pide la copia del acta de nacimiento y la boleta de estudios, para cotejar y validar los documentos originales. Tal proceso se interpreta como un "filtro institucional”, cuyo objetivo principal es comprobar la identidad del alumno (acta de nacimiento) y los grados cursados (boleta de calificaciones). Esto cobra una relevancia especial, porque el estudiante que proviene de cualquier parte de México o del extranjero no cuenta con información capturada en el SICRES, que es estatal, por lo tanto, es necesario comprobar que la información proporcionada por los padres o tutores sea veraz.

Durante el trabajo de campo, a los directores se les preguntó sobre los motivos por los cuales consideraban que las familias migrantes regresan a México, en algunos casos su retorno pudo ser obligado o involuntario. Se les hizo el siguiente cuestionamiento: ¿cuál es el procedimiento que las autoridades escolares siguen en caso de que los 
padres de familia no presenten documentos para inscribir al alumno? Las respuestas fueron heterogéneas, lo cual evidencia la falta de un protocolo institucional aplicable a todos los casos similares (véase Figura 2).

Figura 2. Procedimiento realizado por el director cuando un alumno no presenta documentos para inscribirse

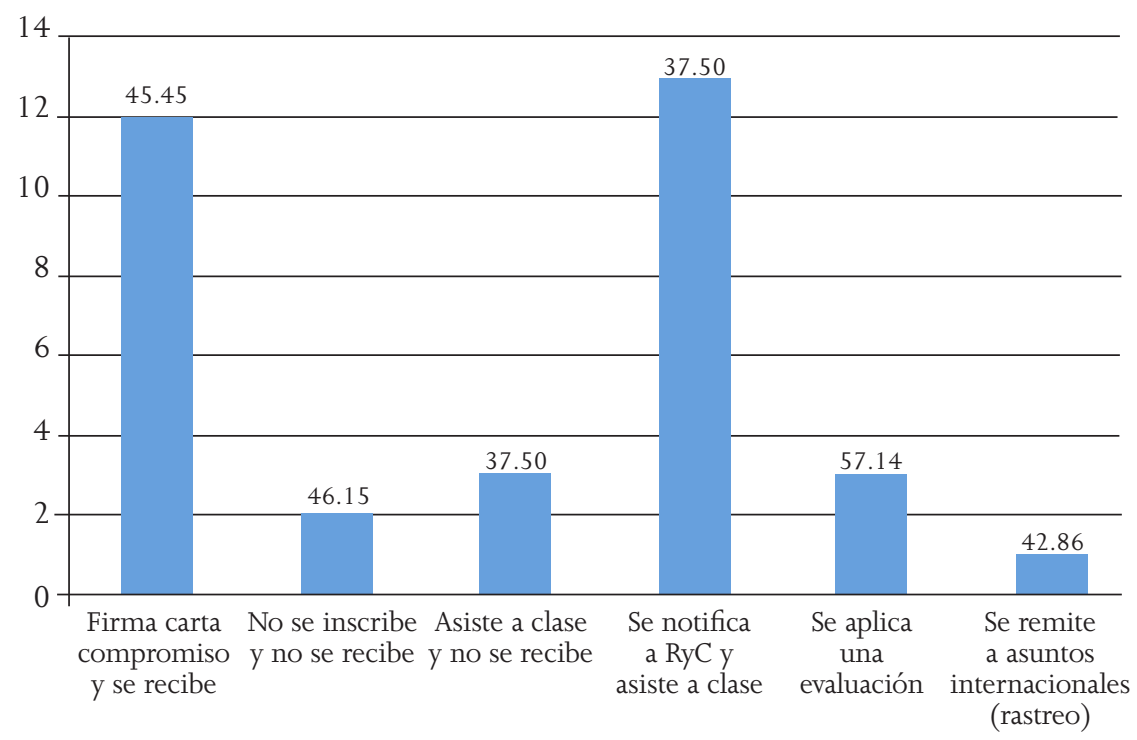

* RyC: Dirección de Registro y Certificación de Educación Básica de la SEC.

Fuente: elaboración propia.

La variedad de las respuestas muestra la ausencia de un protocolo para asistir y atender a una población de alumnos que se encuentra en un estado de vulnerabilidad, como son los/as niñas que en su retorno migratorio no tuvieron oportunidad de recuperar sus documentos escolares o de identidad. Se puede inferir que no existe un proceso estandarizado que considere los sucesos y complicaciones que enfrenta una familia cuando es repatriada o deportada a México. Pareciera que el trabajo del director se limita a canalizar la situación de manera institucional; 13 de los 34 entrevistados expresaron que el 
procedimiento es notificar a la Dirección de Registro y Certificación de Educación Básica para que se encargue de solucionar el problema, mientras los alumnos asisten a la escuela sin estar registrados en el sistema:

Son como niños que están y no están; están en la escuela pero no existen en el sistema como alumnos. Vienen a clases, se les toma asistencia pero no vale porque las asistencias se registran en el portal de SICRES $[\ldots]$ ¿cómo vas a registrarlas [sus asistencias y actividades] si el niño no existe en el sistema? (Director de escuela primaria).

El hecho de que un alumno "esté y no esté" en el sistema implica una diferenciación con el resto del alumnado. Sí asiste a clases, pero nominalmente no es parte del estudiantado, por lo tanto la institución educativa no es consecuente con el discurso de igualdad.

Son como niños fantasma porque el sistema [SICRES] no me los está reconociendo: si no aparece su nombre aquí [en el portal de SICRES] el niño no aparece en las listas [...] Puede venir, puede asistir, pero se le explica al padre de familia: hasta que no me traiga todos los documentos, su hijo no va a quedar inscrito (director de escuela primaria).

Doce directores comentaron que cuando no se presentan documentos, los padres de familia pueden elaborar una carta compromiso en la que se comprometen a entregarlos en un tiempo determinado, ${ }^{9}$ y eso le permite al/la niña asistir a clases. De nuevo, la inscripción formal a la escuela queda condicionada por la firma de la carta compromiso, la cual implica una "justificación" por la falta de la boleta o el acta. De esta manera, el SICRES obtiene el documento firmado por los padres, donde existe un compromiso para entregar la documentación. Sistemáticamente, el proceso automatizado del SICRES le otorga el "estatus" de registrado al momento de capturar dicha información,

9 El tiempo para solicitar los documentos, mediante la carta compromiso, queda a criterio del director. Puede ser de 15 días a un mes, o antes de que termine el ciclo escolar. 
sin embargo, el de inscrito permanece condicionado. A decir de los directores, que un alumno aparezca como registrado en el SICRES significa lo siguiente:

El alumno puede cursar toda la primaria sin presentar un solo documento, cuando termine el sexto año, el certificado de la escuela, el documento oficial que dice que el alumno cursó, terminó y aprobó su primaria, saldrá con una leyendota [sic], así, que atraviesa toda la boleta y dice: provisional. [El certificado] es un documento oficial, un documento que si viene con esa leyenda, pues no le va a servir [...] Es por eso que les insistimos mucho a los padres que traigan los documentos (director de escuela).

El alumno puede terminar su primaria con el estatus de registrado pero el certificado no le va a servir porque dice 'provisional'. Se trata de un documento oficial que tiene esa leyenda y pues no es válido hasta que solvente el error, hasta que entregue los documentos completos $[\ldots]$ (director de escuela).

Dos de los directores manifestaron que si el padre de familia no presenta documentos, el alumno "no se inscribe y no se recibe". Esto como parte de un proceso para salvaguardar la integridad de los/as niñas:

¿Y si llega a pasarle algo? no sé; algún accidente o qué sé yo. Nosotros como directores tenemos toda la responsabilidad y si el niño/a no está inscrito/a, me van a preguntar ¿por qué no está inscrito? [...] El niño no existe en el sistema y por lo tanto no debería estar en la escuela. Es para protección de los mismos niños (director de escuela).

Tenemos indicaciones de ya no recibir niños oyentes. Antes sí se podía, tú recibías al niño y podías decir que era oyente, que venía pero no estaba inscrito en la escuela [...] Nos hicieron ver que si a un niño oyente le llegaba a pasar algo, ¿qué sé yo?, se llega a accidentar, por ejemplo, la escuela no podría hacerse responsable ya que no es un alumno inscrito, ¿me explico? (Director de escuela). 
Figura 3. Proceso de inscripción desde la perspectiva de los directores escolares

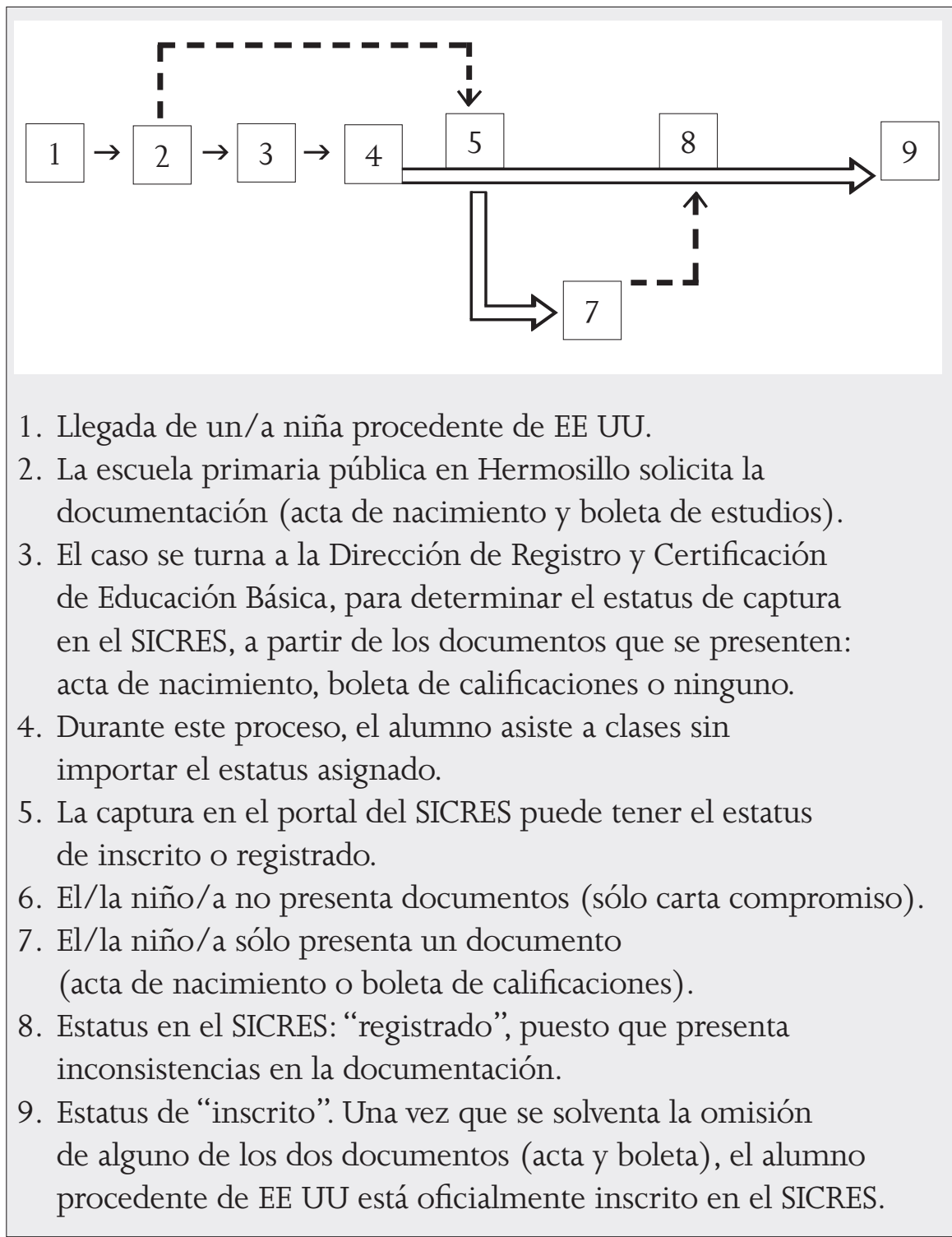

Fuente: elaboración propia.

Para las entrevistas de los directores se diseñó una pregunta relacionada con el tipo de capacitación que han tenido para atender a las familias migrantes que están regresando a México, y solicitan 
inscribir a sus hijos en las escuelas primarias públicas; 77 por ciento señaló no haberla recibido y 21 por ciento que sí. Cuando se les cuestionó acerca de si reciben capacitación específica para los procesos de inscripción de alumnos con experiencia migratoria, respondieron que la SEP, a través de la SEC y la Dirección de Registro y Certificación de Educación Básica llevan a cabo reuniones periódicas y capacitaciones relacionadas con temas generales y en las mesas de trabajo se aborda someramente el tema de niños y niñas migrantes:

[...] muy por encimita [sic]. La capacitación es general; de la Norma ${ }^{10}$ que cada año cambian. De eso es la capacitación que recibimos, pero sí se ha llegado a tocar el tema de los niños migrantes (director de escuela).

En la Figura 3 se presenta el proceso de inscripción que experimentan los niños, las niñas y sus familias. Las flechas punteadas simbolizan las ambigüedades y se intenta describir por pasos los procedimientos que, a partir del discurso de los directores, se deben realizar para lograr una inscripción oficial.

$\mathrm{Al}$ proceso descrito es necesario agregar el contexto situacional de cada familia que está retornando, por ejemplo: el cambio cultural idiosincrásico, el idioma, las instituciones e interacciones sociales disímiles a lo que el/la niña conoce como primera referencia. En este punto el discurso trata a todos los niños por igual, sin distinción alguna generando un choque entre la institución y las familias migrantes de retorno.

\section{Retos del sistema educativo sonorense}

Término académico, institucional y escolar del menor migrante de retorno

Desde hace más de cinco años, hay investigaciones que intentan proponer visiones nuevas y referentes teóricos para categorizar al me-

10 Se refiere a las normas específicas de control escolar relativas a la inscripción, reinscripción, acreditación, promoción, regularización y certificación en la educación básica (SEP 2015). 
nor migrante de retorno. ${ }^{11}$ Este concepto se ha discutido a partir del contexto migratorio de retorno, experimentado por las familias mexicanas retornadas a partir de 2008, cuando enfrentaron la crisis económica y las políticas migratorias restrictivas en EE UU (Valdéz 2012; 2011; Ruiz y Valdéz 2015). En este análisis, el interés principal es construir una definición de menor migrante, desde el contexto integral de la familia mexicana que retorna; se trata de niños y niñas que emigraron a Estados Unidos con su familia o parte de ésta, y que estudiaron en ese país. La referencia también abarca a quienes nacieron allá, cuyos padres son mexicanos, cursaron su educación en escuelas estadounidenses y regresaron a México con los padres. Las investigaciones en Hermosillo, Ciudad Obregón y Nogales han mostrado que la mayoría de los niños que iniciaron su educación en México, y que ahora están de regreso, tienen dificultades al reintegrarse al sistema escolar en las áreas académica, social, cultural y por el idioma. Y los nacidos en EE UU, y que ahora los están trayendo a México, algunos con doble nacionalidad y que nunca habían pisado suelo mexicano, sufren las consecuencias de no hablar ni escribir el español, desconocen las prácticas sociales de convivencia y los contenidos académicos. Además, hay casos en los que los padres permanecen en Estados Unidos, y los niños viven en México sobrellevando las secuelas de la separación y viviendo bajo la tutela de la familia extendida, generalmente con los abuelos (Ruiz y Valdéz 2017). La diversidad de escenarios en los que la familia migrante está de regreso en México es parte de lo que complica la identificación del menor migrante de retorno en el contexto escolar. En la forma de inscripción y acreditación escolar, que se captura por grado a inicio del ciclo, para conocer las estadísticas de los ingresos, la SEP ${ }^{12}$ menciona tres conceptos para referirse al menor migrante de retorno que llega a las aulas: "Alumnos naciona-

11 Cabe aclarar que la perspectiva de análisis que sustenta este trabajo considera el retorno como una consecuencia de los escenarios particulares de la época. Sin embargo, sería prematuro afirmar que se trata de uno permanente, puesto que la migración fluye y se combina con situaciones coyunturales de las sociedades. Es muy probable que las familias que han regresado consideren la temporalidad de su retorno a partir de las condiciones que ofrecen las sociedades mexicana y estadounidense para mejorar su calidad de vida familiar, social y económica.

12 http://www.controlescolar.sep.gob.mx/work/models/controlescolar/Resource/carpeta_ pdf/6_iae_1_2_prim_ind_13_14.pdf 
les provenientes de EE UU con documento de trasferencia, alumnos nacionales provenientes de EE UU sin documento de trasferencia, alumnos provenientes de otros países". Para algunos directores esta terminología resulta confusa, pues comentan que los conceptos "nacionales-provenientes" no aclaran la condición del estudiante, para identificar si es de nacionalidad estadounidense o mexicana. Estos términos tampoco son útiles para identificar si él se inscribe con acta de nacimiento de México o de EE UU, pues casi ninguno presenta el documento de trasferencia, cuando procede de este último; otros directores incluso expresaron desconocer tal documento. La mayoría coincidió en comprender el término "alumnos nacionales provenientes de otros países”, pues entienden que se refiere a los que no son de México ni de Estados Unidos, y que al momento de inscribirse presentaron acta de nacimiento de otro país.

La confusión principal en el uso y llenado de este formato es que los directores desconocen las definiciones y los documentos para identificarlos, por lo que éste no representa las características y la cantidad de menores migrantes de retorno. Por ello se considera fundamental que la SEP integre las definiciones de una manera explícita y contundente, y trasmita esta información a su comunidad escolar, sobre todo a los directores quienes reciben y canalizan a los alumnos. Sin una terminología establecida oficialmente hay ambigüedades en la identificación, el conocimiento, el seguimiento y la evaluación de las poblaciones minoritarias, como los menores migrantes de retorno.

Como ya se expresó, uno de los objetivos de esta investigación fue verificar el número de alumnos migrantes de retorno proporcionado por el PROBEM, a través de sus bases de datos oficiales, y contrastarlo con el reportado por los directores escolares. Se encontraron discrepancias entre los 58 de nuevo ingreso que reportó el PROBEM, durante el ciclo 2014-2015 en las 44 escuelas de Hermosillo, y los 88 que declararon los directores de las 34 escuelas visitadas (véase Figura 4), a quienes ellos identificaron como procedentes de Estados Unidos o con experiencia educativa allá. ${ }^{13}$ Es importante señalar esta

13 Además, a los directores se les preguntó por la cantidad de alumnos procedentes de EE UU o con experiencia en escuelas de allá, inscritos en ciclos pasados. Respondieron que fueron 
diferencia, porque muestra las ambigüedades de los mecanismos de registro e identificación para estudiantes migrantes de retorno que proceden de EE UU, quienes presentan características particulares. Algunos cursaron varios grados en otro estado de México antes de llegar a Hermosillo, por ejemplo, dos hermanos que provenían de una primaria de la ciudad de Chihuahua; estudiaron medio ciclo allá y en el de 2015-2016 solicitaron ingresar a un plantel de Hermosillo. Y, debido a que ellos venían de Chihuahua, no se contabilizaron como procedentes de EE UU, aunque debió ser así para documentar su experiencia educativa y conocer la situación de regulación académica que ello implica al momento de inscribirse en la escuela en México.

Figura 4. Diferencia entre alumnos reportados por el PROBEM y los identificados en trabajo de campo

\begin{tabular}{|c|c|c|c|}
\hline \multicolumn{2}{|c|}{ Escuelas } & $\begin{array}{c}\text { Número de alumnos } \\
\text { reportados por el } \\
\text { PROBEM }\end{array}$ & $\begin{array}{c}\text { Número de alumnos } \\
\text { identificados en trabajo } \\
\text { de campo }\end{array}$ \\
\hline \multirow{3}{*}{ Turno } & Matutino & 36 & 61 \\
\cline { 2 - 4 } & Vespertino & 19 & 27 \\
\cline { 2 - 4 } & Total & 58 & 88 \\
\hline
\end{tabular}

Fuente: elaboración propia.

Método de evaluación inicial: el examen diagnóstico para un menor migrante de retorno

Otro de los hallazgos importantes es el tipo de instrumento de valoración inicial con el que son evaluados los estudiantes procedentes de Estados Unidos. El examen diagnóstico constituye un elemento relevante para identificar los conocimientos previos de todos los estudiantes que se inscriben en las escuelas primarias públicas de

134 los migrantes de retorno en el ciclo 2015-2016; pero no eran ingresos nuevos, sino la matrícula escolar total que a la fecha tenían las 34 escuelas visitadas. 
Hermosillo. Sin embargo, se convierte en un factor de riesgo y ambigüedad para los/a niñas migrantes que provienen de otros sistemas educativos.

Los directores y los profesores deben aplicar una evaluación diagnóstica a todos los estudiantes de nuevo ingreso, incluidos los migrantes, para conocer y satisfacer sus necesidades educativas. Este procedimiento pone en desventaja a los menores migrantes de retorno, ya que desconocen los contenidos del examen debido a su inexperiencia académica en México. Al respecto, los directores comentaron:

Se lleva a cabo una evaluación diagnóstica en cada inicio de ciclo escolar. Es necesario para saber si el niño trae los conocimientos del grado anterior y si no, pues se hacen las adecuaciones necesarias. Todo alumno que inicia un ciclo escolar hace la evaluación diagnóstica. Cuando se trata de un niño que llega a mitad de ciclo, como es el caso de algunos niños migrantes, se hace la evaluación cuando llega; traiga documentos o no los traiga (director de escuela).

Las normas específicas de control escolar relativas a la inscripción, reinscripción, acreditación, promoción, regularización y certificación en la educación básica (SEP 2015) en su artículo 31a, relativo a los criterios aplicables ante la falta de documentos al momento de la reinscripción, en el numeral 31.4, párrafo segundo, expresan la utilidad de este examen:

En el caso de aspirantes a la educación primaria provenientes del extranjero que no presenten el documento del antecedente Escolar de educación preescolar y cumplan con el requisito de la edad señalado en las presentes Normas, el(la) Director(a) de la institución educativa pública o particular con autorización deberá proceder a su inscripción y aplicará una evaluación diagnóstica para determinar las medidas compensatorias que, en su caso, se requieran.

La evaluación diagnóstica, mencionada en dichas normas, es elaborada por los docentes de cada grado escolar, y aplicada a principios 
del ciclo. Algunos directores mencionaron que sólo incluye los temas de español y matemáticas; otros, que también las áreas de ciencias sociales, historia y geografía. Dado que no existe una evaluación diagnóstica estandarizada, elaborada por la SEP o la SEC, los contenidos son diversos. ${ }^{14}$

Cuando se les preguntó a los directores si tenían conocimiento sobre la existencia de una evaluación diagnóstica dirigida y elaborada para un estudiante procedente de Estados Unidos, las respuestas fueron contradictorias. Los resultados muestran que 41 por ciento dijo que sí existía; 38, que no; 15 expresó que se trataba de una evaluación igual para todos, y el resto no tenía información al respecto. Esto refleja la ambigüedad y el desconocimiento de este tipo de procesos administrativos, en especial de 41 por ciento de quienes dijeron conocer una evaluación diagnóstica para dichos alumnos, cuando en realidad tal documento es inexistente.

También se indagó sobre el objetivo de la evaluación, y ocho profesores dijeron que para ubicar al alumno; nueve comentaron que para conocer lo que sabe; 16 señalaron la inexistencia o desconocimiento de una evaluación y uno, que para hacer adecuaciones.

Esto demuestra la falta de información y de capacitación de los directores sobre el tema $y$, por consecuencia, la ausencia de medidas compensatorias y adecuaciones curriculares que permitan evaluar y diagnosticar de manera particular a los niños migrantes de retorno. Por lo que el ingreso de estos menores a las aulas mexicanas carece de un diagnóstico específico y adaptado académicamente a las circunstancias y los saberes previos de un estudiante migrante con experiencia educativa en EE UU, esto en detrimento tanto de su desempeño escolar, debido a la falta de una metodología de evaluación para conocer su nivel de aprendizaje, como de las estrategias de enseñanza diseñadas por el profesor de grupo para darle un seguimiento asertivo y puntual a sus capacidades académicas -aunado a las competencias sociales, que también le competen él-.

14 A partir del ciclo 2015-2016, el Instituto Nacional para la Evaluación de la Educación elaboró una evaluación diagnóstica estandarizada, exclusiva para 4to grado, para el resto el profesor sigue elaborando el examen diagnóstico, que no considera adecuaciones curriculares diferenciadas para ningún tipo de estudiante, sea de otro país o con necesidades educativas especiales. 


\section{Capacitación}

El tema de la capacitación fue uno de los que presentó más incongruencias por parte de los directores. Esto se comprobó con las respuestas obtenidas a dos preguntas planteadas, una acerca de si reciben o no capacitación por parte de la SEP o la SEC, sobre los procesos administrativos de inscripción y sus funciones directivas, cuando se trata de alumnos procedentes de EE UU, y la otra si emiten alguna valoración sobre el servicio de capacitación en dicho proceso. Cerca del 80 por ciento de ellos expresó no recibir capacitación específica sobre estos temas; la estrategia más utilizada es asesorarse directamente con la supervisora escolar, cuando desconocen el procedimiento para la inscripción:

No se toca el tema de los niños migrantes como tal $[\ldots]$ las capacitaciones son de temas generales $[\ldots]$ como son muy pocos los casos [de inscripciones de niños migrantes] pues más bien cuando pasa lo vemos directamente con nuestra supervisora. Conforme se presentan estas situaciones revisamos la Norma o hablamos a Registro y Certificación para que nos digan qué hacer $[\ldots]$ (director de escuela primaria).

Estadísticamente, los ingresos de niños y niñas procedentes de Estados Unidos son poco representativos en la matrícula, sin embargo, es una situación que sucede e incide en el estudiante, su familia y en el contexto escolar en su conjunto. Esto se vuelve relevante y requiere de conocimiento y capacitación apropiada sobre los procesos administrativos y de gestión escolar, pues tanto los trámites como los mecanismos institucionales para incorporar a los menores migrantes a las escuelas debieran ser parte del servicio público del sistema educativo en México, así como ofrecer capacitación específica sobre este tema a los actores educativos a cargo de la inscripción y el registro, sobre todo en las regiones fronterizas como Sonora.

La capacitación se discutió también en las entrevistas con los funcionarios de la SEC quienes, en términos generales, no reconocen que haya una formal sobre este tema. La supervisora comentó que "no hay una capacitación tal cual [...] Registro y Certificación calendariza 
sesiones por zonas escolares a principios de ciclo regularmente. Se ofrecen talleres de actualización en donde pudieran mencionar estos temas, pero de forma muy general”. Los directores dijeron que la función de las supervisoras es de gran apoyo para que "baje" [sic] la información institucional a los centros escolares, tales como acuerdos nuevos, fechas y procedimientos de control escolar; la mayor parte de ésta es proporcionada a los directores por correo electrónico, memorandos o de manera personal por la supervisora.

El coordinador estatal del PROBEM ${ }^{15}$ informó que una o dos veces por ciclo se ofrece capacitación específica a los directores sobre los alumnos migrantes procedentes de EE UU y que también, ocasionalmente, solicitan participar en la que ofrece la Dirección de Registro y Certificación de Educación Básica. Así mismo, comentó que a estas sesiones se invita a todos los directores de las ciudades con mayor representación de este tipo de alumnos, como lo son Nogales, San Luis Río Colorado, Cananea, Cajeme, Navojoa y Hermosillo. El funcionario explicó que desde 2009 el PROBEM brinda capacitación -en la modalidad de taller-donde se presentan, de manera puntual y específica, los procesos administrativos para inscribir y atender a los alumnos procedentes de EE UU; se exponen las situaciones más frecuentes en relación con los documentos presentados y las características de una familia migrante de retorno. El coordinador señaló que del universo de directores convocados sólo asiste entre 20 y 30 por ciento, y que no tiene conocimiento de algún tipo de penalización para los que no se presenten, como tampoco de un incentivo para quienes sí lo hagan. También dijo que desde el ciclo 2011-2012 estas capacitaciones no se ofrecen por falta de presupuesto.

Es relevante mencionar que en trabajo de campo se identificó a los directores de nuevo ingreso. Su contratación es resultado del examen de oposición 2014-2015, donde se asignaron plazas directivas en todo el estado. Poco más de 20 por ciento de éstos sólo ha desempeñado su función durante un ciclo, es decir, desde 2014; el resto de los entrevistados mencionó tener al menos dos ciclos en el cargo. Esto es importante porque muchos de los directores, al no recibir capaci-

15 El coordinador entrevistado fue sustituido a principios de 2016, cuando el Partido Revolucionario Institucional asumió la gubernatura de Sonora. 
tación explícita sobre los estudiantes migrantes, usan su experiencia en situaciones pasadas para tomar decisiones. Por lo tanto, es preocupante pensar cómo procederán en este mismo escenario los de nuevo ingreso, que carecen de capacitación.

Otro aspecto significativo tiene que ver con el mecanismo de captura y resguardo de la información, que tiene la plataforma del SICRES. Los directores comentaron que en ésta se encuentra registrada la información personal del alumno, así como los datos de contacto de los padres y su registro previo en otras escuelas del estado. Y que existe un apartado donde los profesores de grupo hacen observaciones académicas sobre los estudiantes, por ejemplo las dificultades que tienen en algún área social o pedagógica en particular, acuerdos establecidos en conjunto con los padres, o especificaciones importantes sobre las estrategias de enseñanza-aprendizaje más convenientes para ellos.

Algunos directores, de manera voluntaria, a través de sus computadores personales, mostraron el manejo del SICRES: las observaciones académicas capturadas por los profesores de grupo, así como la información estadística de los alumnos del ciclo escolar anterior ya no aparecían en el sistema, a lo que ellos comentaron que todo se borra y se reinicia en cada ciclo. Algunos opinaron que el formateo se pudiera deber a la cantidad exorbitante de memoria o almacenamiento que esta información representa para el SICRES, sin embargo, resulta valiosa para la construcción de estrategias académicas y adecuaciones pertinentes para los alumnos. Además, es una herramienta que apoya la labor docente favoreciendo el trabajo colaborativo entre los profesores. Ante esto, la pregunta que surge es ¿cómo un profesor o director de nuevo ingreso puede darle seguimiento a un estudiante, en particular a uno procedente de EE UU, cuando todo el historial de ciclos previos ha sido borrado?

A todos los directores y al personal administrativo de las instancias de la SEC se les preguntó si en el SICRES existe -para fines de control estadístico y pedagógico- algún término o modalidad que identifique a los estudiantes migrantes de retorno procedentes de EE UU. Para tal pregunta se utilizaron los siguientes términos: alumnos mexicanos, alumnos procedentes de Estados Unidos, alumnos binacionales, 
alumnos migrantes, alumnos regulares ${ }^{16}$ u otro; 22 respondieron que en el SICRES no existe alguna modalidad o clasificación para hacerlo; 5, que se captura bajo la modalidad de "alumno extranjero"; 3 , que se registra como "procedente de EE UU" y sólo uno comentó que se captura como "alumno migrante". Es sorprendente lo desinformados que están los directores sobre el tema, y la facilidad con la que sortean sus respuestas, cuando perciben que están siendo evaluados y confrontados. La realidad es que, como resultado del análisis, se descubrió que en el SICRES no existe una terminología para identificar a los menores migrantes de retorno procedentes de Estados Unidos. Aunque si bien en la norma escolar se utiliza el concepto de "alumno procedente de EE UU” y “alumno extranjero”, la pregunta es ¿de qué sirve entonces incluir estos términos en la norma escolar si en el sistema de captura y registro escolar (SICRES) no existen?

\section{Consideraciones finales}

La llegada de migrantes de retorno procedentes de EE UU a las escuelas mexicanas es un tema complejo, porque el proceso de ingreso, registro e inscripción oficial al sistema de educación es complicado, por la falta de protocolos específicos de normatividad institucional y de procesos normativos y directivos de acompañamiento. Aunado a que existe mucha información aislada, contradictoria y sin un sustento regulado entre los responsables del ingreso. Sin duda, todo este proceso es sensible para quienes lo viven y lo estudian, pues se descubre que son alumnos que están al margen de instrumentos académicos apropiados, que les permitan una transición progresiva y favorable a su nuevo entorno educativo en México. Los hallazgos, en su conjunto, permiten ver una especie de "limbo académico" en donde los menores migrantes de retorno no están situados en las capacitaciones formales, los exámenes estandarizados, ni como una población minoritaria dentro de la escuela. Lo anterior revela que el sistema de

16 Con este concepto, los docentes y directivos se refieren a los alumnos que cursan en tiempo y forma sus estudios de acuerdo con su edad, y sin antecedentes de reprobación o deserción. 
educación no cuenta con una estructura pedagógica e institucional para atender integralmente a esta comunidad.

El alumno migrante de retorno con experiencia académica en EE UU es una categoría que pone en evidencia la falta de prácticas más inclusivas en el sistema educativo, al tiempo que omite las medidas compensatorias necesarias para cada conjunto diferenciado de estudiantes. El sistema y su personal asumen que todos son iguales y que merecen recibir una educación de calidad; tal como los directores lo expresan, "los niños [migrantes] son igual que todos, no hay distinción. Se les considera alumnos normales como a todos $[\ldots]$ ”. Esto tiene sentido desde el discurso institucional, donde todos reciben educación por igual, sin embargo, el reto no es sólo considerar sus semejanzas con el resto, sino identificar sus características contextuales, sociales y académicas propias, a fin de comprender y atender sus necesidades, lo que en definitiva contribuirá a su desarrollo académico desde la parte institucional y pedagógica, pues tan importante se vuelve el proceso de transición durante la llegada a la escuela, como la construcción de nuevos mecanismos para el reconocimiento de sus competencias.

Como se mencionó en la introducción, el artículo emana del proyecto de investigación "Estudio cualitativo sobre características académicas y socioculturales de estudiantes migrantes de retorno a escuelas primarias en el noroeste de México"; aún se analizaban los últimos hallazgos del trabajo de campo; al respecto, se presentaron algunos temas de interés que serán abordados en investigaciones futuras o en otros artículos, como discutir la necesidad de dar seguimiento y retroalimentación efectiva a la plataforma del SICRES, pues dada la información que se captura, este sistema se vuelve importante para el control estadístico de los ingresos, los egresos y las características particulares de los estudiantes. Se sugiere que dicha plataforma integre un concepto específico que permita identificar a los menores migrantes de retorno, con la finalidad de conocer su situación familiar y académica, para estar en condiciones de diseñar herramientas que atiendan sus necesidades. En la actualidad dicha especificación no existe en el sistema, por lo que una de las propuestas de esta investigación es que así como hay un apartado para capturar especificaciones académicas de los estudiantes, sea posible integrar otro en donde se 
identifique si proceden de Estados Unidos o tienen experiencia académica en dicho país. Esto auxiliará a los directores y a los profesores en la identificación y el asesoramiento de los estudiantes. Además, se deben ampliar las categorías de identificación para otros perfiles de alumnos, que requieran adecuaciones educativas más focalizadas. Otra tarea pendiente es exponer más argumentos para la construcción de un término específico que pueda integrarse en los formatos oficiales de la SEP, esto les permitirá a directores, supervisores escolares y profesores tener un control más certero de la cantidad de estudiantes migrantes de retorno procedentes de EE UU, que llegan a las aulas. Como resultado del trabajo de campo y la triangulación de la información obtenida en las entrevistas a directores y funcionarios, se identificaron discursos ambiguos y contradictorios en relación con lo que se entiende por un estudiante procedente de EE UU, un alumno migrante de retorno, un estudiante extranjero o un alumno nacional procedente de ese país. Esta terminología es confusa para los directores, lo cual muestra un campo de oportunidad para los departamentos de capacitación de la SEC, a fin de solventar las dudas y las posibles inconsistencias estadísticas que esto genera.

El retorno migratorio de familias completas o parte ellas también es un campo de estudio que es necesario considerar en los procesos administrativos de inscripción y la incorporación de los alumnos migrantes de retorno a las escuelas de Sonora. Durante esta investigación y trabajo de campo se descubrió que, en algunos casos, son los abuelos, posiblemente los tutores de los/las niñas, quienes los inscriben. Este aspecto se hace notar debido a que el procedimiento en sí mismo resulta complejo para quienes laboran en el sistema de educación -profesores, directores y funcionarios-, y podría resultar aún más para los abuelos o la familia extendida que se hace cargo de los menores durante la tramitología y el ingreso a las escuelas. Los estudiosos de la migración de retorno saben que este proceso no es homogéneo para ninguna de las familias, por lo que es importante considerar al tipo de población que está regresando y enfrentándose a los trámites confusos y por lo regular excesivamente burocratizados del sistema de educación pública.

En definitiva, todavía hay mucho que exponer sobre la migración de retorno y los procesos escolares de ingreso para estudiantes proce- 
dentes de Estados Unidos. Hoy más que nunca es imperativo avanzar en esta discusión, ya que la situación política actual en ese país indica que es muy probable que en el futuro cercano más niños y jóvenes mexicanos en edad escolar regresen a México, como consecuencia de las políticas migratorias racistas y discriminatorias del presidente Donald Trump. El sistema educativo mexicano debe mejorar, a fin de que los menores migrantes de retorno puedan transitar de manera exitosa e incorporarse a él, y su experiencia educativa esté libre de obstáculos administrativos y burocráticos. Se espera que esta investigación contribuya a los estudios de la niñez migrante de retorno en México, y brinde insumos a los estudiosos de temas educativos y a quienes gestionan políticas públicas y ayudan a mejorar los procesos gubernamentales en materia de educación.

\section{Bibliografía}

Cortez Román, Nolvia, Arelys K. García Loya y Adriana Altamirano Ruiz. 2015. Estudiantes migrantes de retorno en México. Estrategias emprendidas para acceder a una educación universitaria. Revista Mexicana de Investigación Educativa 20 (67): 1187-1208.

David, A. Graham. 2016. Has Trump kept his campaign promises? As the president marks 100 days in office, a comprehensive review of his progress toward fulfilling the pledges he made on the trail. The Atlantic. https://www.theatlantic.com/politics/archive/2017/04/trump-promises-cheat-sheet/507347/

Durand, Jorge. 2004. Ensayo teórico sobre la migración de retorno. El principio del rendimiento decreciente. Cuadernos Geográficos 35 (2): 103-116.

Franco García, Martha Josefina. 2014. Los estudiantes inmigrantes: sujetos emergentes del derecho a la educación. Revista Latinoamericana de Estudios Educativos 44 (1): 93-131. 
Gomez, Alan. 2017. Immigration arrests up 38\% nationwide under Trump. USA Today. https://www.usatoday.com/story/news/ world/2017/05/17/deportations-under-president-trump-undocumented-immigrants/101786264/

Kitroeff, Natalie y Shan Li. 2017. Trump's promise to ramp up deportations spreads fear among California businesses. Los Angeles Times. http://www.latimes.com/business/la-fi-immigrants-economicimpact-20170223-story.html

Moctezuma L., Miguel. 2013. Retorno de migrantes a México. Su reformulación conceptual. Papeles de Población 19 (77): 49-160.

Ruiz, Liza F. 2011. Niñez migrante de retorno: el proceso administrativo de inscripción en las escuelas primarias de Hermosillo, Sonora. Tesis de maestría en ciencias sociales, El Colegio de Sonora (COLSON).

Ruiz, Liza F., y Gloria C. Valdéz Gardea. 2017. Sonora en la migración internacional: menores migrantes de retorno. Entre la reforma educativa y los procesos institucionales estatales. En Tránsito y retorno de la niñez migrante. Epílogo en la administración Trump, coordinado por Gloria Ciria Valdéz Gardea e Ismael García Castro, 145-173. Hermosillo: COLSON y Universidad Autónoma de Sinaloa (UAS).

Ruiz, Liza F. y Gloria C. Valdéz Gardea. 2015. El presente educativo en la frontera norte: menores migrantes de retorno y su educación. En Migración y violencia: dos caras del dolor social, coordinado por José Dionicio Vázquez Vázquez, 61-72. Tlaxcala: El Colegio de Tlaxcala.

Ruiz, Liza F. y Gloria C. Valdéz Gardea. 2012. Menores de retorno. El proceso administrativo de inscripción en las escuelas sonorenses. En Movilización, migración y retorno de la niñez migrante. Una mirada antropológica, compilado por Gloria Ciria Valdéz Gardea, 177-214. Hermosillo: COLSON y UAS.

Sánchez García, Juan y Víctor Zúñiga. 2010. Trayectorias de los alumnos transnacionales en México. Propuesta intercultural de atención 
educativa. Trayectorias XII (30): 5-23. <http://www.redalyc.org/articulo.oa?id=60713488002>

SEP. 2015. Normas específicas de control escolar relativas a la inscripción, reinscripción, acreditación, promoción, regularización y certificación en la educación básica. http://www.controlescolar.sep.gob.mx/work/models/controlescolar/Resource/carpeta_ pdf/normas_especificas_basica.pdf (octubre 2015).

Suárez-Orozco, Marcelo y Desiree Qin-Hilliard (editores). 2004. Globalization, culture and education in the new millenium. Berkely: University of California Press y Ross Institute.

Silva Quiroz, Yolanda y Rodolfo Cruz Piñeiro. 2013. Niñez migrante retornada de Estados Unidos por Tijuana: los riesgos de su movilidad. región y sociedad 25 (58): 29-56. DOI: 10.22198/rys.2013.58. a123

Valdéz Gardea, Gloria Ciria. 2012. Movilización, migración y retorno de la niñez migrante. Una mirada antropológica. Hermosillo: COLSON y UAS.

Valdéz Gardea, Gloria Ciria. 2011. La Antropología de la migración: niños y jóvenes migrantes de la globalización. Hermosillo: COLSON y UAS.

Valdéz Gardea, Gloria Ciria e Ismael García Castro (coordinadores). 2017. Tránsito y retorno de la niñez migrante. Epílogo en la administración Trump. Hermosillo: COLSON y UAS.

Valdéz Gardea, Gloria Ciria, Liza Fabiola Ruíz y Óscar Bernardo Rivera. 2017. Avance de investigación. Consecuencias académicas en niños expuestos a la migración internacional: hacia un discurso crítico de los procesos administrativos en el sistema escolar de Sonora, México. Clivajes. Revista de Ciencias Sociales 8: 111-147.

Warren, Robert y Donald Kerwin. 2017. Mass deportations would impoverish US families and create immense social costs. Journal on 
Migration and Human Security V (1): 1-8. http://jmhs.cmsny.org/index.php/jmhs/article/view/71 (febrero 2017).

Ye Hee Lee, Michelle. 2015. Donald Trump's false comments connecting Mexican immigrants and crime. TheWashington Post. https://www. washingtonpost.com/news/fact-checker/wp/2015/07/08/ donald-trumps-false-comments-connecting-mexican-immigrants-and-crime/?utm_term $=.7920$ efb76d58

Zúñiga, Víctor. 2013. Migrantes internacionales en las escuelas mexicanas: desafíos actuales y futuros de política educativa. Revista Electrónica Sinéctica 40: 1-12.

Zúñiga, Víctor, Edmund T. Hamann y Juan Sánchez García. 2008. Alumnos transnacionales: las escuelas mexicanas frente a la globalización. México: SEP. 